Rev. Biol. Trop., 47(4): 697-709, 1999

www.ucr.ac.cr www.ots.ac.cr www.ots.duke.edu

\title{
Distribución, mortalidad y asociación con plantas, de nidos de Paraponera clavata (Hymenoptera: Formicidae) en la isla de Barro Colorado, Panamá
}

\author{
Rolando Pérez, Richard Condit y Suzanne Lao
}

Centro de Ciencias Forestales del Trópico, Instituto de Investigaciones Tropicales Smithsonian, Apartado 2072, Balboa, Rep. de Panamá. Tel. (507) 227-6022 ext. 2346, Fax. (507) 232-5978, Correo electrónico: ctfs@tivoli.si.edu

Recibido 22-V-1998. Corregido 15-VI-1999. Aceptado 16-VI-1999.

\begin{abstract}
We studied the distribution, mortality and association with plants, of 308 nests of the neotropical ant Paraponera clavata, in a permanent 50 hectare plot in old-growth forest on Barro Colorado island between September 1993 and February 1995. Ant nests were uniformly dispersed throughout the plot, and significantly associated with the high plateau and patch of young forest in the plot. The average density was 6.2 nests per hectare. Mortality of nests was higher with increasing number of neighbors within $20 \mathrm{~m}$, compared to those separated at greater distances. The mortality was between $13.36 \%$ and $69.64 \%$ depending on the census interval, and recruitment between $22.63 \%$ and $31.72 \%$. The nests were found in 84 plant species of 34 families, pertaining to four life forms: 76 species were trees, 5 were shrubs, 2 were palms and one was a liana. We tested for association between ant nests and tree species and tree size by examining whether nests were more common in certain categories than would be expected by change. Eight species of plants were positively associated with Paraponera clavata. The ant preferentially selected trees between 8 and $63.9 \mathrm{~cm}$ diameter at breast height. Trees and small shrubs were not associated with the nests. No association was found between ant nests and trees with extra-floral nectaries. Fifty-three percent of the nests had a Phrynus gervaisii (Amblypygi: Phrynidae) cohabiting inside. These nests had lower mortality rates than the rest.
\end{abstract}

Key words: Paraponera clavata, nests, distribution, mortality, recruitment, association, extra-floral nectaries.

Paraponera clavata Smith, 1958 (Hymenoptera: Formicidae: Ponerinae) es una gigantesca $(26 \mathrm{~mm})$ hormiga neotropical que se encuentra distribuida desde Brasil (Sao Paulo) y Paraguay hasta la costa atlántica de Nicaragua (Janzen y Carroll 1983). Esta hormiga es un insecto social que vive en colonias de unos 500 a 1500 individuos (McCluskey y Brown 1972, Janzen y Carroll 1983), construye nidos subterráneos en la base de árboles, palmas y lianas (Belk et al. 1989, Holldobler y Wilson 1990a). P. clavata es omnívora, se alimenta de néctar y pequeños artrópodos como escarabajos, larvas de lepidópteros y arañas (Young y Hermann 1980). Muchos autores (Hermann 1975, Janzen y Carroll 1983, Breed y Bennett 1985, Barrett 1985), observaron a esta hormiga recolectando néctar de los nectarios extraflorales de Pentaclethra macroloba (Leguminosae) y en las inflorescencias de Costus spp. (Zingiberaceae). Es por ello que estos autores han tratado de establecer una relación de asociación no obligatoria entre $P$. clavata y árboles con nectarios. 
La distribución de los nidos de estas hormigas está influida por actividades territoriales de colonias vecinas de la misma especie (Holldobler y Wilson 1990b, Ryti y Case 1992). A escala local, la competencia es un factor de regulación en las poblaciones de hormigas (Holldobler y Lumsden 1980, Holldobler y Wilson 1990b). Las colonias de hormigas pueden compararse con organismos sésiles, porque una vez establecidas, permanecen fijas en un espacio dado y sobreviven por mucho tiempo (Thurber et al. 1993).

Se evalua la distribución, mortalidad y asociación con plantas de nidos de P. clavata en una parcela de cincuenta hectáreas de la isla de Barro Colorado, para responder las siguientes preguntas: ¿Cómo están distribuidos los nidos en la parcela? ¿Cómo son las tasas de mortalidad y reclutamiento general? ¿Hay evidencias de asociación entre los nidos de la hormiga y las plantas de la parcela? ¿Sí los nidos están asociados con árboles con nectarios extraflorales? ¿Sí existen otros organimos que pueden cohabitar en el nido con estas hormigas?

\section{MATERIALES Y MÉTODOS}

Zona de estudio: La isla de Barro Colorado está localizada entre los $9^{\circ} 09^{\prime} \mathrm{N}$ y $79^{\circ}$ 51 ' W. Es una colina de 1500 hectáreas que sobresale $137 \mathrm{~m}$ del lago Gatún en el centro del Canal de Panamá. Croat (1978) y Leigh et al. (1990) describen detalladamente el clima, la flora y la fauna de la isla. Nuestro estudio se hizo en una parcela permanente de cincuenta hectáreas, la cual es un rectángulo de $1000 \mathrm{~m}$ de largo por $500 \mathrm{~m}$ de ancho localizada en la meseta central de la isla. El sitio es relativamente plano, con unas 25 hectáreas de 0-3 por ciento de pendientes, 13 hectáreas de 3-10 por ciento de pendientes, y 10 hectáreas en pendientes moderadas (10-21 por ciento) hacia el este y el sur. También hay un pequeño pantano estacional aproximadamente de 2 hectáreas en la parte central, y una hectárea de bosque joven que bordea el extremo noreste de la parce- la (Hubbell y Foster 1986). El resto del bosque de la parcela es bosque viejo con más de 500 años de edad y pocas perturbaciones (Piperno 1990). Hay un mapa de la parcela en el cual se tienen perfectamente localizados, enumerados con placas de aluminio e identificados, todos los árboles, arbustos y palmas que tienen $1 \mathrm{~cm}$ o más de DAP (diámetro a la altura del pecho $=1.30 \mathrm{~m}$ desde el suelo) (Hubbell y Foster 1983, 1987, Condit et al. 1995). Hasta 1990, se conocía un total de 313 especies de plantas (Condit et al. 1995).

Metodología del muestreo: Entre septiembre de 1993 y febrero de 1995, censamos y marcamos todos los nidos de $P$. clavata en la parcela mediante cuatro censos: dos en la estación lluviosa y dos en la estación seca. Las fechas de los censos fueron: censo 1 (12-17 de septiembre 1993), censo 2 (12-17 de febrero 1994), censo 3 (12-17 de septiembre 1994) y censo 4 (12-17 de febrero 1995). Los nidos fueron censados por columnas de $20 \times 500 \mathrm{~m}$, orientadas de sur a norte y de una hectárea de tamaño. Dentro de cada columna buscamos los nidos por cuadrantes de $20 \times 20 \mathrm{~m}$ siguiendo siempre el mismo patrón. Los nidos encontrados en la base de árboles, arbustos y palmas con más de $1 \mathrm{~cm}$ de DAP, se perturbaron con la ayuda de un tubo de aluminio de $1 \mathrm{~m}$ de largo y $2 \mathrm{~cm}$ de ancho para verificar si estaban ocupados por hormigas. Con la ayuda de la base de datos del Proyecto de Dinámica del Bosque buscamos la identificación, la localización y el DAP de la planta en 1990. Hicimos anotaciones sobre los árboles que presentaron contrafuertes y tenían nectarios extraflorales. Después del primer censo consideramos muertos los nidos visitados que no presentaron hormigas.

Análisis: Estudiamos los patrones de dispersión de los nidos considerando los tamaños de cuadrantes que varían de $20 \times 20 \mathrm{~m}$ hasta 250 x $250 \mathrm{~m}$ usando el índice de dispersión de Morisita (Morisita 1959, Stiteler y Patil 1981). La mortalidad anual la calculamos empleando la fórmula de Condit et al. (1995). Para establecer la relación entre mortalidad y densidad de los nidos, utilizamos la regresión logística múltiple (Engelman 1990), empleando para 
ello las siguientes variables: número de nidos vecinos entre $0-10 \mathrm{~m}$ del nido, entre $10-20 \mathrm{~m}$, entre 20-30 m y entre 30-40 m. Determinamos el reclutamiento anual utilizando la siguiente fórmula: $r=100[\operatorname{In}(S+R)-\operatorname{In}(S)] / t$, donde: $r=$ reclutamiento anual en porcentaje, $S=$ número de nidos que sobreviven entre el primer y segundo censo, $R=$ reclutas en el segundo censo, $t=i n t e r v a l o$ en años. Para analizar la asociación de los nidos con las especies de plantas de la parcela de cincuenta hectáreas, comparamos la abundancia total de nidos observada con la esperada, tomando en cuenta el número total de individuos de las especies de plantas en la parcela y empleando los datos más actualizados del censo de 1990. Aquellas especies de plantas con relativamente pocos individuos (menos de 800), se agruparon y analizaron en conjunto de acuerdo a su forma de vida.

\section{RESULTADOS}

Distribución de los nidos: Entre septiembre de 1993 y febrero de 1995, se encon- tró 308 nidos de $P$. clavata en una parcela de cincuenta hectáreas de la isla de Barro Colorado (Fig. 1). Se registró 237 nidos en el primer censo, 196 en el segundo, 202 en el tercero y 210 en el cuarto, la densidad promedio fue de 6.2 nidos por hectárea. Los resultados obtenidos con el índice de Morisita (Cuadro 1) indican que los nidos están distribuidos uniformemente (Fig. 1), guardando cierta distancia uno del otro, especialmente en el caso de cuadrantes de $20 \times 20 \mathrm{~m}$ y $25 \times 25 \mathrm{~m}$, donde el índice es menor de 1 . Parece ser que los nidos se asocian de manera significativa con el tipo de hábitat. Encontramos que existen más nidos de los esperados en las planicies altas y especialmente en la sección de bosque joven de la parcela (Cuadro 2), en comparación con otras áreas.

Mortalidad y reclutamiento de los nidos: La mortalidad de nidos es alta, (69.64\%) 60 nidos murieron en un intervalo de cinco meses entre el primer y segundo censo, $(26.54 \%)$ 28 en un intervalo de siete meses entre el segundo y tercero, y $(13.36 \%) 11$ en un intervalo de cinco meses entre el tercero y el cuarto. Los

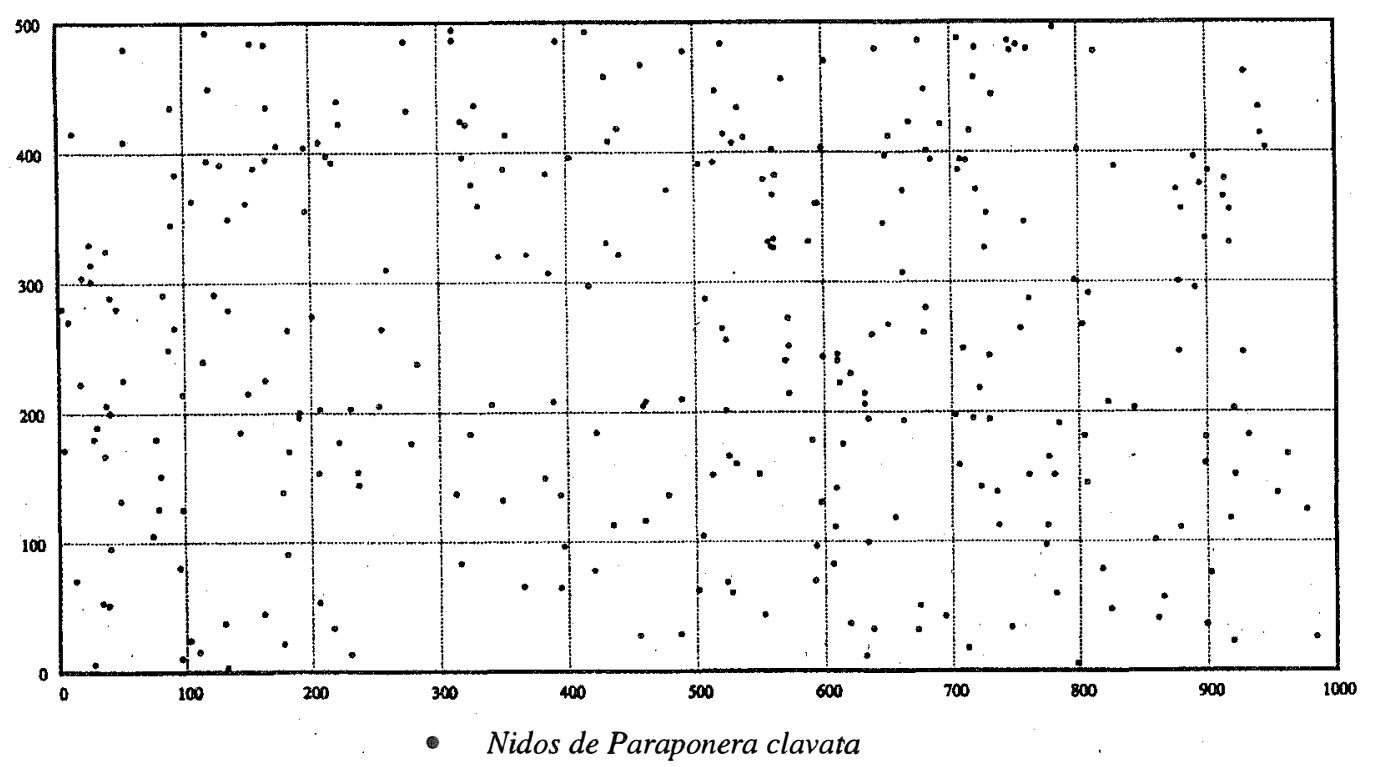

Fig. 1. Distribución de los nidos de P. clavata en la parcela de cincuenta hectáreas de la iṣla de Barro Colorado Fig. 1. Distribution of the P. clavata nests in the Barro Colorado Island 50 hectare plot 


\section{CUADRO 1}

Patrones de dispersión de los nidos de Paraponera clavata en la parcela de cincuenta hectáreas de la isla de Barro Colorado utilizando el índice de dispersión de Morisita.

Dispersion patterns of the Paraponera clavata nest in Barro Colorado Island 50 ha plot using Morisita's index.

$\begin{array}{lllll}\text { Censo } & \begin{array}{l}\text { Tamaño del } \\ \text { cuadrante en }(\mathrm{m})\end{array} & \begin{array}{l}\text { Número de } \\ \text { cuadrantes }\end{array} & \text { Número de nidos } & \begin{array}{l}\text { Indice de } \\ \text { Morisita }\end{array} \\ 1 & 20 \times 20 & 1250 & & \\ 1 & 25 \times 25 & 800 & 236 & 0.5860 \\ 1 & 40 \times 40 & 300 & 236 & 0.6635 \\ 1 & 50 \times 50 & 200 & 236 & 0.9831 \\ 1 & 100 \times 100 & 50 & 236 & 0.9737 \\ 1 & 200 \times 200 & 10 & 189 & 0.9629 \\ 1 & 250 \times 250 & 8 & 236 & 0.9873 \\ 2 & 20 \times 20 & 1250 & 196 & 0.0135 \\ 2 & 25 \times 25 & 196 & 0.3768 \\ 2 & 40 \times 40 & 187 & 0.9315 \\ 2 & 50 \times 50 & 196 & 0.0152 \\ 2 & 100 \times 100 & 200 & 196 & 0.9890 \\ 2 & 200 \times 200 & 50 & 156 & 0.0108 \\ 2 & 250 \times 250 & 10 & 196 & 1.0792 \\ 3 & & 8 & & 0.4926 \\ 3 & 20 \times 20 & 1250 & 202 & 0.4729 \\ 3 & 25 \times 25 & 202 & 0.8653 \\ 3 & 40 \times 40 & 194 & 0.9064 \\ 3 & 50 \times 50 & 300 & 202 & 0.9630 \\ 3 & 100 \times 100 & 200 & 202 & 0.9998 \\ 3 & 200 \times 200 & 50 & 163 & 1.0612 \\ 4 & 250 \times 250 & 10 & 202 & 0.2848 \\ 4 & 20 \times 20 & 8 & 210 & 0.2187 \\ 4 & 25 \times 25 & 1250 & 210 & 0.7839 \\ 4 & 40 \times 40 & 800 & 200 & 0.8111 \\ 4 & 50 \times 50 & 300 & 210 & 0.9501 \\ 4 & 100 \times 100 & 200 & 169 & 1.0031 \\ 4 & 200 \times 200 & 50 & 210 & 1.0411 \\ & 250 \times 250 & 10 & & \end{array}$

\section{CUADRO 2}

Distribución de los nidos de Paraponera clavata con respecto al tipo de hábitat en la parcela de cincuenta hectáreas de la isla de Barro Colorado.

Distribution of the Paraponera clavata nests according to habitat in Barro Colorado Island 50 ha plot.

$\begin{array}{lcccccc}\text { Hábitat } & \begin{array}{c}\text { Cantidad } \\ \text { de } 5 \times 5 \mathrm{~m}\end{array} & \begin{array}{c}\text { \% en la } \\ \text { parcela }\end{array} & \text { Observado } & \text { Esperado } & \begin{array}{c}\text { Chi } \\ \text { cuadrado }\end{array} & \begin{array}{c}\text { Densidad } \\ \text { nidos/has. }\end{array} \\ \text { Planicie alta } & 2993 & 14.97 & 61 & 46.11 & 4.81 & 8.2 \\ \text { Planicie baja } & 10669 & 53.35 & 168 & 164.32 & 0.08 & 6.3 \\ \text { Pendiente } & 4805 & 24.02 & 56 & 73.98 & 4.37 & 4.7 \\ \text { Pantano } & 678 & 3.39 & 2 & 10.44 & 6.82 & 1.2 \\ \text { Bosque nuevo } & 855 & 4.27 & 21 & 13.18 & 4.64 & 9.8 \\ \text { Total } & 20000 & 100 & 308 & 308.03 & 20.72 & 6.2\end{array}$

Grados de libertad $=4$; probabilidad $<0.01$ 
análisis muestran que el número de nidos vecinos entre $0-10 \mathrm{~m}$ o entre $10-20 \mathrm{~m}$ de distancia influyen en la sobrevivencia, mientras más vecinos hay a esa distancia, mayor es la probabilidad de mortalidad para los nidos; sin embargo, el número de nidos vecinos entre 20-30 m y 30-40 $\mathrm{m}$ no influyen en la sobrevivencia (Fig. 2). Hubo (24.34\%) 19 reclutas (nidos nuevos de la hormiga) entre el primer y segundo censo, $(31.72 \%) 34$ entre el segundo y tercero, y $(22.63 \%) 19$ entre el tercero y el cuarto.
Asociación de nidos con plantas: Los nidos estaban en la base de 84 especies de plantas, en 34 familias con cuatro categorías de forma de vida: 76 especies eran árboles, 5 especies eran arbustos, 2 especies eran palmas y 1 era liana (Cuadro 3). Solamente 8 especies (9.52\% del total) tuvieron nectarios extraflorales, y no se encontró asociación entre tales especies y $P$. clavata $\left(\mathrm{X}^{2}=0.000886 ; \mathrm{P}<0.976\right)$. Utilizando todos los censos juntos 8 especies de plantas estuvieron asociadas positivamente

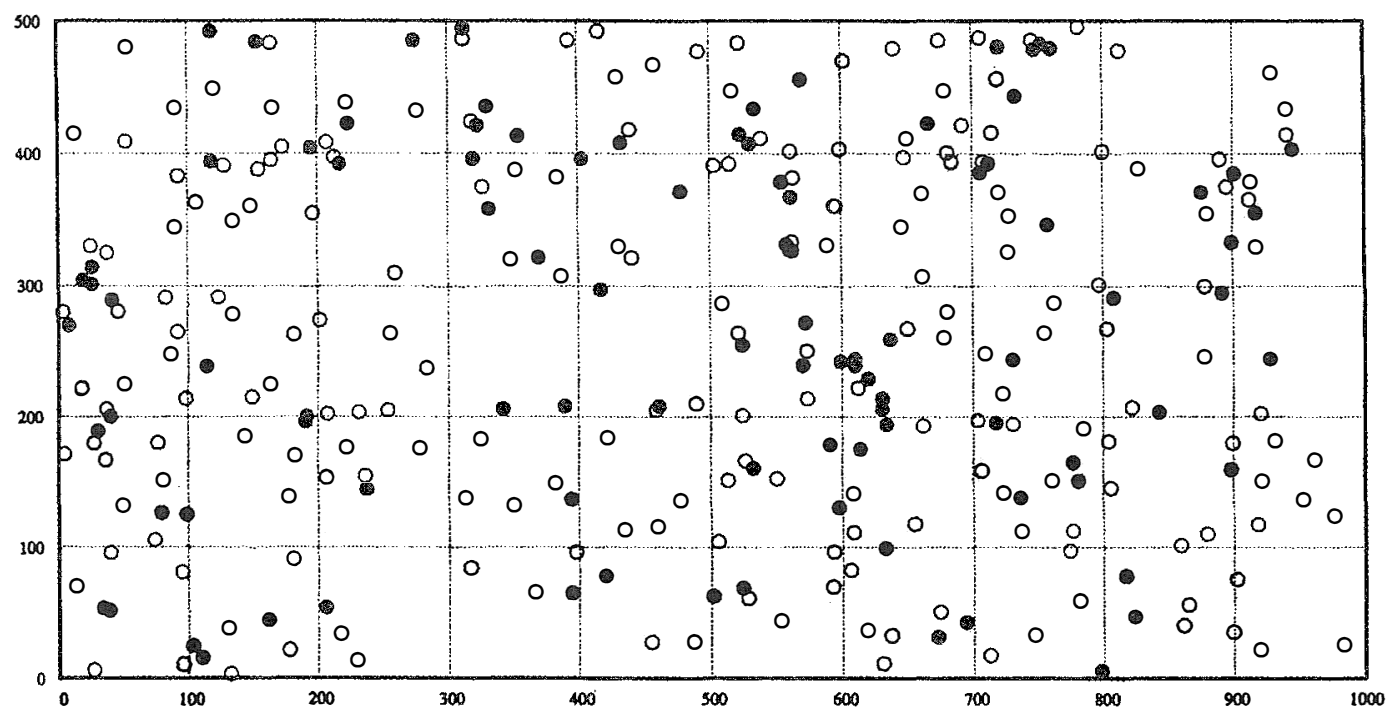

Nidos vivos de Paraponera clavata

Nidos muertos de Paraponera clavata

Fig. 2. Distribución de la mortalidad de los nidos de P. clavata en la parcela de cincuenta hectáreas en la isla de Barro Colorado

Fig. 2. Distribution of the mortality of the P. clavata nests in the Barro Colorado Island 50 hectare plot

con los nidos de P. clavata (Cuadro 4); y 3 especies presentaron asociación negativa con los nidos (Cuadro 5). En el análisis en conjunto de las especies de plantas menos comunes en la parcela (menos de 800 individuos), el grupo de arbustos tenían significativamente menor cantidad de nidos de $P$. clavata que lo esperado basado en su abundancia colectiva en la parcela $\left(\mathrm{X}^{2}=5.0801 ; \mathrm{P}<0.050\right)$, mientras que el grupo de árboles grandes tenían significativamente más nidos que lo esperado $\left(\mathrm{X}^{2}=\right.$ 123.2641; $\mathrm{P}<0.005$ ). Plantas entre 8 y $63.9 \mathrm{~cm}$ en tamaño de DAP son las que $P$. clavata utiliza con mayor frecuencia para establecer los nidos (Cuadro 6). Utilizando sólo los árboles dentro de esta clase de tamaño, 5 especies de 


\section{CUADRO 3}

Especies y familias de plantas con nidos de Paraponera clavata en la parcela de cincuenta hectáreas de la isla de Barro Colorado. Se muestran también las especies que tuvieron nectarios extraflorales ++ y la cantidad de individuos de plantas con contrafuertes.

Plant species and family with Paraponera clavata nests in Barro Colorado Island 50 ha plot. Species with extra-floral nectaries and the number of trees with buttresses are also shown.

Especies

Alchornea costaricensis

Alseis blackiana

Apeiba membranacea

Aspidosperma cruenta

Beilschmiedia pendula

Calophyllum longifolium

Capparis frondosa

Cecropia insignis

Ceiba pentandra

Chrysophyllum argenteum

Cordia alliodora

Cordia bicolor

Cordia lasiocalyx

Croton billbergianus

Cupania latifolia

Cupania sylvatica

Dendropanax stenodontus

Desmopsis panamensis

Diospyros arthantifolia

Drypetes standleyi

Entada monostachya *

Eugenia galalonensis

Faramea occidentalis

Ficus obtusifolia

Ficus tonduzii

Garcinia intermedia

Guapira standleyanum

Guarea guidonia

Guarea sp.

Guettarda foliacea

Gustavia superba

Hasseltia floribunda

Heisteria concinna

Hirtella triandra

Hybanthus prunifolius

Inga quaternata

Inga ruiziana

Inga sapindoides

Jacaranda copaia

Laetia thamnia

Licania hypoleuca

Lonchocarpus latifolius

Luehea seemannii

Macrocnemum glabrescens

Maquira costaricana

Nectandra cissiflora

Nectandra globosa

Nectandra purpurea
Familia

Forma de vida

Euphorbiaceae

Rubiaceae

Tiliaceae

Apocynaceae

Lauraceae

Guttiferae

Capparidaceae

Moraceae

Bombacaceae

Sapotaceae

Boraginaceae

Boraginaceae

Boraginaceae

Euphorbiaceae

Sapindaceae

Sapindaceae

Araliaceae

Annonaceae

Ebenaceae

Euphorbiaceae

Leguminosae

Myrtaceae

Rubiaceae

Moraceae

Moraceae

Guttiferae

Nyctaginaceae

Meliaceae

Meliaceae

Rubiaceae

Lecythidaceae

Flacourtiaceae

Olacaceae

Chrysobalanaceae

Violaceae

Leguminosae

Leguminosae

Leguminosae

Bignoniaceae

Flacourtiaceae

Chrysobalanaceae

Leguminosae

Tiliaceae

Rubiaceae

Moraceae

Lauraceae

Lauraceae

Lauraceae

Arbol

Arbol

Arbol

Arbol

Arbol

Arbol

Arbol

Arbol

Arbol

Arbol

Arbusto

Arbol

Arbol

Liana

Arbol

Arbol

Arbol

Arbol

Arbol

Arbol

Arbol

Arbol

Arbol

Arbol

Arbol

Arbol

Arbol

Arbusto

Arbol

Arbol

Arbol

Arbol

Arbol

Arbol

Arbol

Arbol

Arbol

Arbol

Arbol

Arbol

Arbol
Número de nidos

Con nectarios

Con contrafuertes 
Especies

\begin{abstract}
Familia
\end{abstract}
Palmae

Piperaceae

Moraceae

Moraceae

Sapotaceae

Leguminosae

Burseraceae

Burseraceae

Rubiaceae

Leguminosae

Bombacaceae

Rubiaceae

Euphorbiaceae

Monimiaceae

Palmae

Malpighiaceae

Anacardiaceae

Guttiferae

Bignoniaceae

Apocynaceae

Leguminosae

Combretaceae

Flacourtiaceae

Burseraceae

Burseraceae

Meliaceae

Meliaceae

Myristicaceae

Myristicaceae

Myristicaceae

Annonaceae

Rutaceae

Rutaceae

Rutaceae Forma de vida
número de
nidos

Con nectarios

Con contrafuertes

Arbol
Palma
Arbusto
Arbol
Arbol
Arbol
Arbol
Arbol
Arbol
Arbusto
Arbol
Arbol
Arbol
Arbol
Arbol
Palma
Arbol
Arbol
Arbol
Arbol
Arbol
Arbol
Arbol
Arbol
Arbol
Arbol
Arbol
Arbol
Arbol
Arbol
Arbol
Arbol
Arbol
Arbol
Arbol

Arbol
3

Total $=84$

Nota: * se eliminó en algunas de las calculaciones, debido a que no se tienen datos de lianas en la parcela.

\section{CUADRO 4}

Especies de plantas asociadas positivamente con los nidos de Paraponera clavata en la parcela de cincuenta hectáreas de la isla de Barro Colorado.

Plants species positively associated with Paraponera clavata nests in the Barro Colorado Island 50 ha plot.

$\begin{array}{lcc}\text { Especies } & \text { Número de nidos } & \text { Chi-cuadrado } \\ \text { Oenocarpus mapora } & 51 & 1044.09^{* *} \\ \text { Tabernaemontana arborea } & 10 & 33.41^{* *} \\ \text { Protium tenuifolium } & 11 & 11.54^{* *} \\ \text { Virola sebifera } & 8 & 9.14^{* *} \\ \text { Poulsenia armata } & 8 & 8.81^{* *} \\ \text { Guarea guidonia } & 7 & 6.63^{*} \\ \text { Protium costaricensis } & 4 & 5.30^{*} \\ \text { Beilschmiedia pendula } & 8 & 4.79^{*}\end{array}$

Grados de libertad $=1 ; * *$ probabilidad $<0.005 ; *$ probabilidad $<0.050$ 


\section{CUADRO 5}

Especies de plantas asociadas negativamente con los nidos de Paraponera clavata en la parcela de cincuenta hectáreas de la isla de Barro Colorado.

Plant species negatively associated with Paraponera clavata nests in the Barro Colorado Island 50 ha plot.

$\begin{array}{lcc}\text { Especies } & \text { Número de nidos } & \text { Chi-cuadrado } \\ \text { Hybanthus prunifolius } & 5 & 48.59^{* *} \\ \text { Desmopsis panamensis } & 2 & 11.26^{* *} \\ \text { Psychotria horizontalis } & 1 & 4.86^{*} \\ \text { Grados de libertad }=1 ;{ }^{* *} \text { probabilidad }<0.005 ;{ }^{*} \text { probabilidad }<0.050 & \end{array}$

plantas resultaron positivamente asociadas con los nidos: Oenocarpus mapora $\left(\mathrm{X}^{2}=108.3002\right.$; $\mathrm{P}<0.005)$, Virola surinamensis $\left(\mathrm{X}^{2}=20.6851\right.$; $\mathrm{P}<0.005)$, Tabernaemontana arborea $\left(\mathrm{X}^{2}=\right.$ 14.8250; $\mathrm{P}<0.005)$, Protium tenuifolium $\left(\mathrm{X}^{2}=\right.$ $11.5081 ; \mathrm{P}<0.005)$ y Cecropia insignis $\left(\mathrm{X}^{2}=\right.$ $7.1800 ; \mathrm{P}<0.010)$; y Faramea occidentalis $\left(\mathrm{X}^{2}\right.$ $=10.8537 ; \mathrm{P}<0.005)$ presentó asociación negativa con los nidos; pero esta planta cae en los límites inferiores de tamaño dentro de la clase.

Interacción con otros animales: De los 308 nidos que censamos (53\%) 163 tenían el amblipígido Phrynus gervaisii (Pocock) (Amblypygi: Phrynidae) habitando en su interior. Se realizaron análisis de mortalidad tomando como base la presencia del amblipígido en los nidos registrados en el primer censo, y cuántos de ellos habían muerto para el cuarto censo. Los resultados muestran que los nidos donde habían amblipígidos tenían una menor tasa de mortalidad (17\%), en comparación con aquellos donde no hubo amblipígidos (61\%).

\section{CUADRO 6}

Distribución observada y esperada de las clases de tamaños de plantas usadas por Paraponera clavata para fabricar sus nidos.

Observed and expected distribution by diameter size classes of the plants used by Paraponera clavata to build their nests.

Clases de tamaños de
DAP en $(\mathrm{cm})$

$\begin{array}{ccc}1-1.9 & 9 & 119.48 \\ 2-3.9 & 10 & 100.67 \\ 4-7.9 & 28 & 49.8 \\ 8-15.9 & 68 & 22.56 \\ 16-31.9 & 122 & 9.73 \\ 32-63.9 & 57 & 3.84 \\ 64< & 13 & 0.92\end{array}$

Nidos esperados

19.48

22.56

3.84

0.92 


\section{DISCUSIÓN}

Distribución de los nidos: La densidad era de 6.2 nidos con hormigas por hectárea, un poco diferente a 4.34 nidos por hectárea encontrados por Thurber et al. (1993), y $4.0 \mathrm{ni}$ dos por hectárea en 3.5 hectáreas (Breed y Bennett 1985) en Costa Rica. Los cálculos del índice de Morisita (Cuadro 1) en donde calculamos el índice para cada uno de los censos, muestran que los nidos no estaban distribuidos aleatoriamente, sino uniformemente, guardando cierta distancia uno del otro, esta condición ocurrió cuando el índice era menor de 1 y se mantuvo a través de todos los censos. Esto nos indica que las colonias de $P$. clavata compiten por el territorio. Sin embargo, el índice aumenta con el tamaño de los cuadrantes, hasta llegar a un índice mayor de 1, ésto ocurrió en áreas mayores que $250 \times 250 \mathrm{~m}$, en la cual la uniformidad se hace difusa, si la comparamos con cuadrantes más pequeños.

Los nidos se asociaban de manera significativa con el tipo de hábitat y encontramos que existían más nidos de los esperados en las planicies altas y en la sección de bosque joven de la parcela. En lugares altos y planos los nidos se protegen mejor de las lluvias, ya que el agua fluye por escorrentía desde estos sitios hasta la base de las pendientes, en donde los nidos pueden ser afectados por las inundaciones. La escasa cantidad de nidos en el área del pantano (Cuadro 2), que permanece inundado durante gran parte de la estación lluviosa, es una buena evidencia de lo mencionado anteriormente. También existían más nidos de los esperados en la sección de bosque joven, en el borde nororiental de la parcela. En este área existían muchas lianas que la hormiga emplea para ir a muchos lugares en busca de alimento (McCluskey y Brown 1972, Hermann 1975). Belk et al. (1989), reporta la presencia de nidos en la base de lianas, pero no señala en cuales especies se encontraban. El único nido encontrado por nosotros en la base de una liana estaba en Entada monostachya (Leguminosae), que es la liana más grande que alcanza el dosel del bosque en la isla de Barro Colorado (Croat
1978), y que se extiende en gran parte de la región del bosque joven de la parcela. También existe en esta sección de la parcela una alta densidad de árboles de Gustavia superba (Lecythidaceae). En reiteradas ocasiones se observó los individuos de $P$. clavata recolectando néctar en las flores de esta planta, y en una ocasión en las flores de la liana Clitoria javitensis (Leguminosae).

Mortalidad y reclutamiento de los nidos: Los análisis señalan que la mortalidad era más alta en aquellos nidos que tienen un mayor número de vecinos entre $0-10 \mathrm{~m}$ y entre $10-20 \mathrm{~m}$ de distancia, mientras que vecinos a más de 20 $m$ no parecen influir en la mortalidad de los nidos. Thurber et al. (1993), señala que la agresión entre colonias vecinas es una de las principales causas de mortalidad de $P$. clavata. En nuestro caso esto suele ocurrir con frecuencia en las planicies altas y en la sección de bosque joven de la parcela, en donde existe una mayor densidad de nidos y las hormigas compiten por el territorio (Fig. 2). En varias ocasiones observamos el enfrentamiento entre colonias vecinas, durante el combate, muchas obreras salen de los nidos cargando larvas y capullos, y sobre ellas vuelan moscas parásitas de Apocephalus paraponerae (Diptera: Phoridae) que parasitan las obreras heridas de $P$. clavata (Brown y Feener 1991). Esto provoca cambios en el comportamiento normal de la hormiga, pero no se sabe hasta qué punto puede afectar o contribuir con la mortalidad de los nidos. Muchos nidos murieron entre el primer y segundo censo (septiembre 1993-febrero 1994), septiembre, octubre y noviembre son los meses de mayor precipitación en la isla (Windsor et al. 1990), lo cual posiblemente afecta el establecimiento y mortalidad de los nidos. Sugerimos realizar estudios más detallados al respecto. Hubo un mayor número de reclutas o nidos que aparecieron entre el segundo y tercer censo (febrero 1994-septiembre 1994), finales de la estación seca y comienzo de la lluviosa. Foster (1990), señala que existe un pico de floración en la isla poco después de iniciarse la estación lluviosa, el cual coincide con la abundancia máxima de insectos. Los árboles maduros producen flores y néctar, 
lo cual atrae a su vez muchos insectos polinizadores. Estas son fuentes de alimento para P. clavata. Una mayor disponibilidad de alimento probablemente puede explicar mucho sobre la actividad y la aparición de nidos nuevos de estas hormigas. En reiteradas ocasiones se observaron en los nidos formas reproductivas (hembras y machos alados) entre julio y noviembre. Wilson (1971), establece que para que aparezcan las formas aladas en una colonia de hormigas ésta debe contar con un suplemento de alimento adecuado, debido a que la demanda energética aumenta.

Asociación de nidos con plantas: Se encontró un gran número de especies de plantas empleadas por $P$. clavata para fabricar sus nidos. Sin embargo, el gran número, 84 especies de 34 familias de plantas, nos sugiere que existe muy poca selección de la hormiga por las especies de plantas que selecciona para fabricar sus nidos. Del total de plantas con nidos, sólo 8 especies (9.52\%) presentaron nectarios extraflorales, muy diferente de $23 \%$ encontrado por Belk et al. (1989) en un estudio similar; sin embargo estos autores presentan en su listado de árboles con nectarios plantas que carecen de ellos como: Ceiba pentandra (Bombacaceae), Ouratea lucens (Ochnaceae), Hirtella triandra (Chrysobalanaceae), Gustavia superba (Lecythidaceae), Simarouba amara (Simaroubaceae), Terminalia amazonia (Combretaceae), Guarea guidonia, Guarea sp. y Trichilia tuberculata (Meliaceae). Breed y Bennett (1985), señalan una asociación positiva entre $P$. clavata y Pentaclethra macroloba (Leguminosae), un árbol grande con nectarios extraflorales muy común en la estación La Selva en Costa Rica, el cual no existe en la isla de Barro Colorado. En la parcela de cincuenta hectáreas de la isla existen alrededor de 28 especies $(2.73 \%$ del total de individuos), que son árboles grandes que tienen nectarios y pueden ser una buena alternativa para la hormiga. Un buen ejemplo lo constituye el género Inga (Leguminosae), que tiene en la parcela 14 especies (1.72\% del total de individuos). Sin embargo, sólo 3 especies de Inga, con 1 individuo cada una, tuvieron nidos, y no se encontró asociación entre tales especies y $P$. clavata.
Todas las especies de plantas asociadas positivamente con los nidos de $P$. clavata son árboles del dosel medio y alto, y los que presentan asociación negativa son arbustos pequeños. Oenocarpus mapora (Palmae), una palma del dosel medio que tiene $0.73 \%$ del total de individuos en la parcela (Cuadros 3 y 4), es la planta de mayor asociación con los nidos de $P$. clavata. Los individuos de $O$. mapora crecen en pequeños grupos condensados (clones) (Croat, 1978), cada grupo está compuesto de 5 a 15 tallos, que se entrelazan con otros árboles y lianas que ascienden a distintos niveles del dosel, lo cual favorece que la hormiga pueda salir a muchos lugares en busca de alimento. Las numerosas hojas de esta planta, conjuntamente con su sistema radical, que se encuentra ligeramente elevado de la superficie del suelo, deben proteger mejor los nidos de $P$. clavata de las lluvias, las inundaciones y los mantienen ocultos de los depredadores. Además, los nidos establecidos en la base de estas palmas permanecen en un lugar húmedo y sombreado, y quizás pueden soportar mejor las altas temperaturas de la estación seca.

Las hormigas prefirieron plantas entre $8 \mathrm{y}$ $63.9 \mathrm{~cm}$ de DAP (Cuadro 6), esto posiblemente es una evidencia de asociación entre estas plantas y los nidos. En la parcela existen $11.51 \%$ del total de individuos de plantas entre estas clases de tamaño de DAP, y en ellas registramos 242 nidos $(78.8 \%$ del total). La menor cantidad de nidos $21.7 \%$, la encontramos en plantas que presentaban un DAP entre 1 y $7.9 \mathrm{~cm}$ o por encima de $64 \mathrm{~cm}$ (Cuadro 6). La mayoría de las plantas de la parcela (88.48\% del total de individuos) se encuentran en los diámetros señalados, y en ellas encontramos la menor cantidad de nidos. En las plantas entre 8 y $63.9 \mathrm{~cm}$ de DAP: Oenocarpus mapora, Virola surinamensis, Tabernaemontana arborea y Protium tenuifolium, se encontraron asociadas con los nidos de $P$. clavata, lo cual coincide con los resultados del (Cuadro 4). Posiblemente estas plantas son las que ofrecen las mejores oportunidades para el establecimiento de los nidos en la parcela de cincuenta hectáreas. 
Los resultados obtenidos señalan que existe una asociación entre los nidos de la hormiga y las plantas. Sin embargo, hemos observado recientemente algunos árboles que tuvieron nido en el pasado que están muertos y otros que caen vivos desde su base, esto nos sugiere que los nidos de esta hormiga pueden ocasionar daños a las plantas a largo plazo.

Interacción con otros animales: Muchos insectos y otros animales habitan en los nidos de las hormigas y entre ellos hay depredadores, parásitos, huéspedes indiferentes y huéspedes verdaderos (Holldobler y Wilson 1990b). $P$. gervaisii es un inquilino oportunista de los nidos de $P$. clavata (LeClerc et al. 1987). Pareciera ser que $P$. gervaisii reconoce los nidos de $P$. clavata que se encuentran más adaptados para sobrevivir y los emplea como un refugio durante un período prolongado de tiempo. El amblipígido emerge del nido junto con las obreras de $P$. clavata al perturbar el nido, y por lo general, su presencia parece pasar inadvertida por las obreras. Sólo observamos a las hormigas atacarlo en una ocasión. Durante el combate una obrera introdujo el aguijón varias veces en su cuerpo ocasionándole la muerte, y luego lo abandonó cerca de la entrada del nido. La historia natural de los amblipígidos es poco conocida (Quintero 1981), sin embargo P. gervaisii se encuentra habitando un gran número de nidos de $P$. clavata y se reproduce dentro de los nidos entre julio y octubre, y en muy pocas ocasiones en marzo; por lo que resultaría interesante estudiar si existe una posible asociación.

Se demuestra muchas de las características importantes de la distribución, mortalidad y asociación con plantas, de los nidos de la hormiga tropical Paraponera clavata en una parcela de cincuenta hectáreas de bosque viejo en la isla de Barro Colorado, Panamá. Que las ubicaciones precisas de 308 nidos de la hormiga sean conocidas, provee una base de información que puede ser ventajosa para una gran variedad de investigaciones a corto y largo plazo. Esto puede comprender los campos de la ecología reproductiva, variaciones estacionales, impactos ocasionados por cambios climáticos sobre la pobla- ción, y las interacciones y asociaciones con otros animales. Tales investigaciones brindarían en el futuro las bases para un mejor conocimiento sobre la biología de la especie.

\section{AGRADECIMIENTOS}

Agradecemos a Stephen Hubbell y Robin Foster por sus trabajos en el establecimiento de la parcela de cincuenta hectáreas de la isla de Barro Colorado. A Diomedes Quintero por la identificación del especímen de Phrynus ger vaisii. A Raúl Rios por facilitarnos información de la precipitación en la isla de Barro Colorado. A Michael Kaspari, Leeanne Alonso y sonathan González, por los comentarios sobre el manuscrito. Al Instituto de Investigaciones Tropicales Smithsonian y todo su personal de trabajo en la isla de Barro Colorado, por las facilidades que nos brindaron durante la investigación.

\section{RESUMEN}

Se estudió la distribución, mortalidad, reclutamiento y asociación con plantas, de 308 nidos de la hormiga neotropical Paraponera clavata en una parcela de cincuenta hectáreas de bosque viejo en la isla de Barro Colorado entre septiembre de 1993 y febrero de 1995. Los nidos estaban distribuidos uniformemente a través de toda la parcela, y se asociaban de manera significativa con el tipo de hábitat, existían más nidos de los esperados en las planicies altas y en la sección de bosque más joven de la parcela. La densidad promedio era de 6.2 nidos por hectárea. Los nidos con un mayor número de vecinos entre 0 y $20 \mathrm{~m}$ de distancia, tenían una mayor probabilidad de morir, si se comparaban con aquéllos que se encontraban separados por distancias mayores. La mortalidad era entre 13.36 y $69.64 \%$ dependiendo del intervalo de censo, y el reclutamiento fue del 22.63 y $31.72 \%$. Los nidos se encontraron en las bases de 84 especies de plantas, en 34 familias con cuatro categorías de forma de vida: 76 especies eran árboles, 5 especies eran arbustos, 2 especies eran palmas y 1 era liana. Ocho especies de plantas se asociaban positivamente con los nidos de la hormiga. Plantas medianas entre 8 y $63.9 \mathrm{~cm}$ de DAP eran las más utilizadas. Arboles y arbustos pequeños presentan muy poca asociación con los nidos. La hormiga no se asocia con árboles que tienen nectarios extraflorales. El 53\% de los nidos tenían a Phrynus gervaisii (Amblypygi: Phrynidae) habitando en el interior. Estos nidos presentaron menores tasas de mortalidad en comparación con el resto. 


\section{REFERENCIAS}

Barrett, B.A., C.D. Jorgensen \& S.J. Looman. 1985. Foraging recruitment by the giant tropical ant, Paraponera clavata (Hymenoptera: Formicidae). Pan- Pac. Entomol. 61: 334-338.

Belk, M.C., H. L. Black, C. D. Jorgensen, S.P. Hubbell \& R.B. Foster. 1989. Nest tree selectivity by the tropical ant Paraponera clavata. Biotropica 21: 173-177.

Breed, M.D. \& B. Bennett. 1985. Mass recruitment to nectar sources in Paraponera clavata; field study. Insectes Soc. 32: 198-208.

Brown, B.V. \& D.H. Feener, Jr. 1991. Behavior and host location cues of Apocephalus paraponerae (Diptera: Phoridae), a parasitoid of the giant tropical ant, $\mathrm{Pa}$ raponera clavata (Hymenoptera: Formicidae: Ponerinae). Entomol. News 86: 94-98.

Condit,R., S. P. Hubbell \& R. B. Foster. 1995. Mortality Rates of 205 Neotropical Tree and Shrub Species and the Impact of a Severe Drought. Ecol. Monographs, 65: 419-439.

Croat, T.B. 1978. Flora of Barro Colorado Island. Stanford Univ., Stanford, California. 943p.

Engelman, L. 1990. Stepwise Logistic Regression. p. 1013-1046. In: Dixon, W. J., M. B. Brown, L. Engelman y L. 1. Jennrich (eds.). BMDP Statistical Software Manual. University of California, Berkeley, California.

Foster, R.B. 1990. Ciclo estacional de caída de frutos en la isla de Barro Colorado. 219-241. Leigh, E. Jr.,A. S. Rand y D. M. Windsor (eds.). Ecología de un Bosque Tropical. Ciclos estacionales y cambios a largo plazo. Smithsonian Tropical Research Institute, Balboa, Panamá.

Hermann, H.R. 1975. Crepuscular and nocturnal activities of Paraponera clavata (Hymenoptera: Formicidae: Ponerinae). Entomol. News 86: 94-98.

Holldobler, B. \& C.J. Lumsden. 1980. Territorial strategies in ants. Science 210: 732-739.

Holldobler, B. \& E.O. Wilson. 1990a. Host tree selection by the neotropical ant Paraponera clavata (Hymenoptera: Formicidae). Biotropica 22: 213-214.

Holldobler, B. \& E.O. Wilson. 1990b. The ants. The Belknap of Harvard Univ., Cambridge. 732p.

Hubbell, S.P. \& R.B. Foster. 1983. Diversity of canopy trees in a neotropical forest and implications for con- servation. p. 25-41 In: Sutton, S.L., T.C. Whitmore and A.C. Chadwick (eds.). Tropical.Rain Forest: Ecology and Management. Blackwell Scientific, Oxford.

Hubbell, S.P. \& R.B. Foster. 1986. Commonness and rarity in a neotropical forest: implications for tropical tree conservation. In: M. Soulé (ed.), Conservation Biology: The Science of Scarcity and Diversity. Sinauer, Sunderland, MA, p. 205-231.

Hubbell, S.P. \& R.B. Foster. 1987. La estructura espacial a gran escala de un bosque neotropical. Rev. Biol. Trop. 35: 7-22.

Janzen, D.H. \& C.R. Carroll. 1983. Paraponera clavata. p. 752-753. In: D.H. Janzen (ed.). Costa Rica Natural History. Univ. of Chicago, Chicago.

LeClerc, M.G., D.C. McClain, H.L. Black \& C.D. Jorgensen. 1987. An inquiline relationship between the tailless whip-scorpion Phrynus gervaisii and the giant tropical ant Paraponera clavata. Journal Arachnol. 15: $129-130$.

Leigh, E. G. Jr., S. A. Rand \& M. D. Windsor. 1990. Ecología de un Bosque Tropical. Ciclos estacionales y cambios a largo plazo. Smithsonian Tropical Research Institute, Balboa, Panamá. 546 p.

McCluskey, A.M. \& W.L. Brown. 1972. Rhythms and other biology of the giant tropical ant Paraponera. Psyche 79: 335-347.

Morisita, M. 1959. Measuring of the dispersion of individuals and analysis of the distributional patters. Mem. Fac. Sci., Kyushu Univ., Ser. E. (Biol.), 2: 21 5-235.

Piperno, D.R. 1990. Fitolitos, arqueología y cambios prehistóricos de la vegetación en un lote de cincuenta hectáreas de la isla de Barro Colorado. 153-156. Leigh, E. Jr., A.S. Rand y D.M. Windsor (eds.). Ecología de un Bosque Tropical. Ciclos estacionales y cambios a largo plazo. Smithsonian Tropical Research Institute, Balboa, Panamá.

Quintero, D. 1981. The amlypygi genus Phrynus in the Americas (Amblypygi: Phrynidae). J. Arachnol. 9: 117-166.

Ryti, R.T. \& T.J. Case. 1992. The role of neighborhood competition in the spacing and diversity of ant communities. Amer. Nat. 139: 355-374.

Stiteler, W. \& Patil, G. P. 1981. Variance-to-mean ratio and Morisita's index as measures of spatial patters in ecological populations. In: Statistical Ecology Vol. 1 (ed G.P.Patil, E. C. Pielou and W. E. Waters), p. 423-452. 
The Pensylvania State University, University Park and London.

Thurber, D.H., M.C. Belk, H.L. Black, C.D. Jorgensen, S.P. Hubbell \& R.B. Foster. 1993. Dispersion and mortality of colonies of the tropical ant Paraponera clavata. Biotropica 25: 215-221.

Windsor, M.D., A.S. Rand \& W.M. Rand. 1990. Características de la precipitación de la isla de Barro Colorado. 53-71. Leigh, E. Jr., A.S. Rand y D.M. Windsor (eds.). Ecología de un Bosque Tropical. Ciclos estacionales y cambios a largo plazo. Smithsonian Tropical Research Institute, Balboa, Panamá.

Wilson, E. O. 1971. The Insect Societes. The Belknap of Harvard Univ. Cambridge, 548p.

Young, A.M. \& H.R. Hermann. 1980. Notes of foraging of the giant tropical ant Paraponera clavata (Hymenoptera: Formicidae: Ponerinae). J. Kansas Entomol. Soc. 53: 35-55. 